\title{
The State of the Art in Cross-Layer Design for Wireless Sensor Networks
}

\author{
Tommaso Melodia, Mehmet C. Vuran, and Dario Pompili \\ Broadband and Wireless Networking Laboratory, \\ School of Electrical \& Computer Engineering, \\ Georgia Institute of Technology, Atlanta, GA 30332 \\ \{tommaso, mcvuran, dario\}@ece.gatech.edu
}

\begin{abstract}
The literature on cross-layer protocols, protocol improvements, and design methodologies for wireless sensor networks (WSNs) is reviewed and a taxonomy is proposed. The communication protocols devised for WSNs that focus on cross-layer design techniques are reviewed and classified, based on the network layers they aim at replacing in the classical open system interconnection (OSI) network stack. Furthermore, systematic methodologies for the design of cross-layer solution for sensor networks as resource allocation problems in the framework of non-linear optimization are discussed. Open research issues in the development of cross-layer design methodologies for sensor networks are discussed and possible research directions are indicated. Finally, possible shortcomings of cross-layer design techniques such as lack of modularity, decreased robustness, and instability are discussed, and precautionary guidelines are presented.
\end{abstract}

\section{Introduction}

There exist exhaustive amount of research to enable efficient communication in wireless sensor networks (WSNs) 1. Most of the proposed communication protocols improve the energy efficiency to a certain extent by exploiting the collaborative nature of WSNs and its correlation characteristics. However, the main commonality of these protocols is that they follow the traditional layered protocol architecture. While these protocols may achieve very high performance in terms of the metrics related to each of these individual layers, they are not jointly optimized to maximize the overall network performance while minimizing the energy expenditure. Considering the scarce energy and processing resources of WSNs, joint optimization and design of networking layers, i.e., cross-layer design, stands as the most promising alternative to inefficient traditional layered protocol architectures.

Accordingly, an increasing number of recent papers have focused on the crosslayer development of wireless sensor network protocols. In fact, recent papers on WSNs 13] 18, 31] reveal that cross-layer integration and design techniques result in significant improvement in terms of energy conservation. Generally, there are 
three main reasons behind this improvement. First, the stringent energy, storage, and processing capabilities of wireless sensor nodes necessitate such an approach. The significant overhead of layered protocols results in high inefficiency. Moreover, recent empirical studies necessitate that the properties of low power radio transceivers and the wireless channel conditions be considered in protocol design. Finally, the event-centric approach of WSNs requires application-aware communication protocols.

Although a consistent amount of recent papers have focused on cross-layer design and improvement of protocols for WSNs, a systematic methodology to accurately model and leverage cross-layer interactions is still missing. With this respect, the design of networking protocols for multi-hop wireless ad hoc and sensor networks can be interpreted as the distributed solution of resource allocation problems at different layers. However, while most of the existing studies decompose the resource allocation problem at different layers, and consider allocation of resources at each layer separately, we review recent literature that has tried to establish sound cross-layer design methodologies based on the joint solution of resource allocation optimization problems at different layers.

Several open research problems arise in the development of systematic techniques for cross-layer design of WSN protocols. In this paper, we describe the performance improvement and the consequent risks of a cross-layer approach. We review literature proposing precautionary guidelines and principles for crosslayer design, and suggest some possible research directions. We also present some concerns and precautionary considerations regarding cross-layer design architectures. A cross-layer solution, in fact, generally decreases the level of modularity, which may loose the decoupling between design and development process, making it more difficult to further design improvements and innovations. Moreover, it increases the risk of instability caused by unintended functional dependencies, which are not easily foreseen in a non-layered architecture.

The paper is organized as follows. In Section 2, we overview the communication protocols devised for WSNs that focus on cross-layer design techniques. We classify these techniques based on the network layers they aim at replacing in the classical OSI network stack. Moreover, a new communication paradigm, i.e., cross-layer module, is introduced. In Section 3, we discuss the resource allocation problems that relate to the cross-layer design and the proposed solutions in WSNs. Based on the experience in cross-layering in WSNs, in Section 4 we list the potential open problems that we foresee for WSNs. Then, we stress some reservations about cross-layer design by discussing its pros and cons in Section 5 . and conclude the paper in Section 6 .

\section{Cross-Layer Protocols for Wireless Sensor Networks}

The experience gained through both scientific studies and experimental work in WSNs revealed important interactions between different layers of the network stack. These interactions are especially important for the design of communication protocols for WSNs. 
As an example, in [15], the effect of wireless channel on a simple communication protocol such as flooding is investigated through testbed experiments. Accordingly, the broadcast and asymmetric nature of the wireless channel results in a different performance than that predicted through unit disk graph models (UDG). Similarly, in 38, the experimental studies reveal that the perfectreception-within-range models can be misleading in performance evaluations due to the existence of transitional region in low power links. Moreover, in [26, guidelines for physical-layer-driven protocol and algorithm design are investigated. These existing studies strongly advocate that communication protocols for WSN need to be re-designed considering the wireless channel properties. Similarly, as pointed out in 31, the interdependency between local contention and end-to-end congestion is important to be considered in protocol design. The interdependency between these and other network layers call for adaptive crosslayer mechanisms for efficient data delivery in WSNs.

In addition to the wireless channel impact and cross-layer interactions, spatiotemporal correlation is another significant characteristic of sensor networks. Dense deployment of sensor nodes results in the sensor observations being highly correlated in the space domain. Similarly, the nature of the energy-radiating physical phenomenon yields temporal correlation between each consecutive observation of a sensor node. Exploiting the spatial and temporal correlation further improves energy efficiency of communication in WSNs [30].

Next, we overview representative communication protocols that are relevant to the cross-layering philosophy. Moreover, we overview a single module solution for efficient communication in WSNs.

\subsection{Existing Work}

Cross-layer approach has so far been used in two main context in WSNs. In many papers, the cross-layer interaction is considered, where the traditional layered structure is preserved, while each layer is informed about the conditions of other layers. However, the mechanisms of each layer still stay intact. On the other hand, there is still much to be gained by rethinking the mechanisms of network layers in a unified way so as to provide a single communication module for efficient communication in WSNs. In this section, we also focus on the cross-layer module design, where functionalities of multiple traditional layers are melted into a functional module.

In the following, the literature of WSN protocols with cross-layer principles are surveyed. We classify these studies in terms of interactions or modularity among physical (PHY), medium access control (MAC), routing, and transport layers.

$M A C+P H Y$ : In [17, the energy consumption analysis for physical and MAC layers is performed for three different MAC protocols. The authors provide analysis of energy consumption and conclude that single-hop communication can be more efficient if real radio models are used. Although this is an interesting result, the analysis is based on a linear network, which may not be practical in realistic scenarios. 
A cross-layer solution among MAC layer, physical phenomenon, and the application layer for WSNs is proposed in [32. The spatial correlation in the observed physical phenomenon is exploited for medium access control. Based on a theoretical framework, it is shown that a sensor node can act as a representative node for several other sensor nodes. Accordingly, a distributed, spatial correlation-based collaborative medium access control (CC-MAC) protocol is proposed. Simulation results show that exploiting spatial for medium access results in high performance in terms of energy, packet drop rate, and latency.

$M A C+$ Routing: In many work, the receiver-based routing is exploited for MAC and routing cross-layer modularity. In this approach, the next hop is chosen as a result of the contention in the neighborhood. Receiver-based routing has been independently proposed in [28, 35], and [36]. In [35] and [36, the authors discuss the energy efficiency, latency, and multihop performance of the algorithm. In [37, the work in [35] and [36] is extended for a single radio node. In 28, the receiverbased routing is also analyzed based on a simple channel model and lossless links. Moreover, the latency performance of the protocol is presented based on different delay functions and collision rates. Although the authors provide insightful results for the receiver-based routing, the impact of physical layer is not considered in the protocol operation. Similarly in [14, the routing decision is performed as a result of successive competitions at the medium access level. More specifically, the next hop is selected based on a weighted progress factor and the transmit power is increased successively until the most efficient node is found. Moreover, on-off schedules are used. The performance evaluations of all these propositions present the advantages of cross-layer approach at the routing and MAC layers.

A joint scheduling and routing scheme is proposed in 27. for periodic traffic in WSNs. In this scheme, the nodes form distributed on-off schedules for each flow in the network while the routes are established such that the nodes are only awake when necessary. Since the traffic is periodic, the schedules are then maintained to favor maximum efficiency. The authors also investigate the tradeoff between on-off schedules and the connectivity of the network.

The usage of on-off schedules in a cross-layer routing and MAC framework is also investigated in [18. In this work, a TDMA-based MAC scheme is devised, where nodes distributively select their appropriate time slots based on local topology information. The routing protocol also exploits this information for route establishment. In [18, the authors advocate the usage of cross-layer interaction through comparative simulations with a strict layered approach.

WSNs are characterized by multiple flows from closely located nodes to a single sink. However, if this fact is not considered in route establishment, potential interfering routes can be established. In [13, this effect of broadcast nature of MAC on routing is investigated. In this work, MAC interference between routes is minimized by constructing interference-aware routes. The routes are constructed using node codewords that indicate the interference level of nodes and each packet contains a route indicator for route establishment. As a result, the routes are constructed to minimize the interference among them. 
Routing + PHY: A cross-layer optimization of network throughput for multihop wireless networks is presented in 34. The authors split the throughput optimization problem into two sub-problems, i.e., multi-hop flow routing at the network layer and power allocation at the physical layer. The throughput is tied to the per-link data flow rates, which in turn depend on the link capacities and hence, the per-node radio power level. On the other hand, the power allocation problem is tied to interference as well as the link rate. Based on this solution, a CDMA/OFDM based solution is provided such that the power control and the routing are performed in a distributed manner.

In [25, new forwarding strategies for geographic routing are proposed based on the results in [38. The authors provide expressions for the optimal forwarding distance for networks with automatic repeat request (ARQ) and without ARQ. Moreover, two forwarding strategies for these cases are provided. The forwarding algorithms require the packet reception rate of each neighbor for determination of the next hop and construct routes accordingly. Although the new forwarding metrics illustrate the advantages of cross-layer forwarding techniques in WSNs, the analysis for the distribution of optimal hop distance is based on a linear network structure.

Transport + PHY: In [8], a cross-layer optimization solution for power control and congestion control is considered. The authors provide analytical analysis of power control and congestion control, and the trade-off between layered and cross-layer approach is presented as discussed in Section 3. Based on this framework, a cross-layer communication protocol based on CDMA is proposed, where the transmission power and the transmission rate is controlled. However, the proposed solutions only apply to CDMA-based wireless multihop networks, which may not apply to WSNs that CDMA technology may not be feasible.

3-Layer Solutions: In addition to the proposed protocols that focus on pairwise cross-layer interaction, more general cross-layer approaches among three protocol layers exist. In [22], the optimization of transmission power, transmission rate, and link schedule for TDMA-based WSNs is proposed. The optimization is performed to maximize the network lifetime, instead of minimizing the total average power consumption. In [11, joint routing, MAC, and link layer optimization is proposed. The authors consider a variable-length TDMA scheme and MQAM modulation. The optimization problem considers energy consumption that includes both transmission energy and circuit processing energy. Based on this analysis, it is shown that single-hop communication may be optimal in some cases where the circuit energy dominates the energy consumption instead of transmission energy. Although the optimization problems presented in the paper are insightful, no communication protocol for practical implementation is proposed. Moreover, the transport layer issues such as congestion and flow control are not considered.

\subsection{Cross-Layer Module (XLM) for Wireless Sensor Networks}

The cross-layer approach emerged recently still necessitates a unified cross-layer communication protocol for efficient and reliable event communication that 
considers transport, routing, and medium access functionalities with physical layer (wireless channel) effects for WSNs. Here, we overview a new communication paradigm, i.e., cross-layer module (XLM) for WSNs 3 . XLM replaces the entire traditional layered protocol architecture that has been used so far in WSNs.

The basis of communication in XLM is built on the initiative concept. The initiative concept constitutes the core of XLM and implicitly incorporates the intrinsic functionalities required for successful communication in WSN. A node initiates transmission by broadcasting an RTS packet to indicate its neighbors that it has a packet to send. Upon receiving an RTS packet, each neighbor of a node decides to participate in the communication through initiative determination. Denoting the initiative as $\mathcal{I}$, it is determined as follows:

$$
\mathcal{I}=\left\{\begin{array}{l}
1, \text { if }\left\{\begin{array}{l}
\xi_{\text {RTS }} \geq \xi_{T h} \\
\lambda_{\text {relay }} \leq \lambda_{\text {relay }}^{\text {Th }} \\
\beta \leq \beta^{\text {max }} \\
E_{\text {rem }} \geq E_{\text {rem }}^{\text {min }}
\end{array}\right. \\
0, \text { otherwise }
\end{array}\right.
$$

where $\xi_{R T S}$ is the received SNR value of the RTS packet, $\lambda_{\text {relay }}$ is the rate of packets that are relayed by a node, $\beta$ is the buffer occupancy of the node, and $E_{r e m}$ is the residual energy of the node, while the terms on the right side of the inequalities indicate the associated threshold values for these parameters, respectively. The initiative, $\mathcal{I}$, is set to 1 if all four conditions in (1) are satisfied. The first condition ensures that reliable links be constructed for communication. The second and third conditions are used for local congestion control in XLM. The second condition prevents congestion by limiting the traffic a node can relay. The third condition ensures that the node does not experience any buffer overflow. The last condition ensures that the remaining energy of a node $E_{r e m}$ stays above a minimum value, $E_{\text {rem }}^{\min }$.

The cross-layer functionalities of XLM lie in these constraints that define the initiative of a node to participate in communication. Using the initiative concept, XLM performs local congestion control, hop-by-hop reliability, and distributed operation. For a successful communication, a node first initiates transmission by broadcasting an RTS packet, which serves as a link quality indicator and also helps the potential destinations to perform receiver-based contention. Then, the nodes that hear this initiation perform initiative determination according to (11). The nodes that decide to participate in the communication contend for routing of the packet by transmitting CTS packets. The waiting times for the CTS packet transmission is determined based on the advancement of a node for routing [3]. Moreover, the local congestion control component of XLM ensures energy efficient as well as reliable communication by a two-step congestion control. Analytical performance evaluation and simulation experiment results show that XLM significantly improves the communication performance and outperforms the traditional layered protocol architectures in terms of both network performance and implementation complexity. 


\section{Cross-Layer Resource Allocation}

The design of networking protocols for multi-hop wireless ad hoc and sensor networks can be interpreted as the distributed solution of resource allocation problems at different layers. Resource allocation in the context of multi-hop wireless networks has been extensively studied in the last few years, typically with the objectives of maximizing the network lifetime [6], minimizing the energy consumption [23, and maximizing the network capacity 24]. However, most of the existing studies decompose the resource allocation problem at different layers, and consider allocation of the resources at each layer separately. Resource allocation problems are treated either heuristically, or without considering cross-layer interdependencies, or by considering pairwise interactions between isolated pairs of layers.

A typical example of the tight coupling between functionalities handled at different layers is the interaction between the congestion control and power control mechanisms 8 . The congestion control regulates the allowed source rates so that the total traffic load on any link does not exceed the available capacity. In typical congestion control problems, the capacity of each link is assumed to be fixed and predetermined. However, in multi-hop wireless networks, the attainable capacity of each wireless link depends on the interference levels, which in turn depend on the power control policy. Hence, congestion control and power control are inherently coupled and should not be treated separately when efficient solutions are sought.

Furthermore, the physical, medium access control (MAC), and routing layers together impact the contention for network resources. The physical layer has a direct impact on multiple access of nodes in wireless channels by affecting the interference at the receivers. The MAC layer determines the bandwidth allocated to each transmitter, which naturally affects the performance of the physical layer in terms of successfully detecting the desired signals. On the other hand, as a result of transmission schedules, high packet delays and/or low bandwidth can occur, forcing the routing layer to change its route decisions. Different routing decisions alter the set of links to be scheduled, and thereby influence the performance of the MAC layer.

Several papers in the literature focus on the joint power control and MAC problem and/or power control and routing issues, although most of them study the interactions among different layers under restricted assumptions. In Section 3.1, we report a set of significative examples. In Section 3.2, we describe previous work that dealt with cross-layer design of multi-hop wireless networks within an optimization framework. In Section 3.3, we discuss a general framework for describing cross-layer optimization problems.

\subsection{Related Work: Pairwise Interactions}

In 12, the problem of scheduling maximum number of links in the same time slot is studied. The objective of the paper is to develop a power control based multiple access algorithm for contention-based wireless ad hoc networks, so that the network maximum per-hop throughput is achieved. 
In [10, the problem of joint routing, link scheduling, and power control to support high data rates for broadband wireless multi-hop networks is analyzed. In particular, the work focuses on the minimization of the total average transmission power, subject to given constraints regarding the minimum average data rate per link, as well as peak transmission power constraints per node.

In 20, the joint power control and scheduling problem is addressed under the assumption that the session paths are already given. This work aims at satisfying the rate requirements of the sessions not only in the long term, as considered in [10, but also in the short term, in order to prevent the sessions with low jitter or bounded delay requirement from suffering from the ambiguity of the long term guarantees. The need for close interactions between these layers is demonstrated, and it is pointed out that independent decisions at different layers for achieving a local objective would deteriorate the performance of other layers.

\subsection{Related Work: Optimization Frameworks}

Recent studies, and in particular the pioneering work by Low [21] and Chiang [8], have demonstrated the need to integrate various protocol layers into a coherent framework, to help provide a unified foundation for the analysis of resource allocation problems, and to develop systematic techniques for cross-layer design of multi-hop wireless networks. These results are built on recently developed nonlinear optimization theory for the design of communication systems. In particular, convex optimization [5] and geometric programming [9] optimization techniques have been recently proposed and investigated. The objective is to develop a framework that accurately models every aspect of the layered network architecture, resulting in new theoretical results and in practical new design perspectives.

The main technique used in these papers is the method of dual decomposition for convex optimization problems. The technique of dual decomposition has been used by Low in 21, where parameters describing congestion are interpreted as primal and dual optimization variables, while the TCP protocol is interpreted as a distributed primal-dual protocol solving a distributed utility maximization problem. In [8], transmitted power levels and congestion window sizes are jointly optimized. The amount of bandwidth supplied to the upper layers is nonlinearly coupled to the bandwidth demanded by the congestion control through a dual variable. In [7, the cross-layer design of congestion control, routing, and scheduling are jointly tackled by extending the framework of utility maximization introduced in 8. In [33, a primal-dual method is proposed for distributed solution of a joint source coding, routing, and channel coding. In [34, an optimization framework is proposed that jointly optimizes routing and power allocation by also relying on network coding techniques.

\subsection{A General Framework for Cross-Layer Design Problems}

As discussed above, the current trend is to formulate increasingly complex crosslayer resource allocation problems in multi-hop wireless networks as optimization problems. While details of the model are dependent on the particular problem 
being dealt with, it is possible to outline a general framework where particular problems can fit by specifying the form of particular functions. We build on the framework proposed in 8] and further specify it, for example by including latency bounds. Hence, we introduce the following notations that are used in the general formulation:

$-\mathbf{r}=\left[r_{1}, r_{2}, . ., r_{s}, . ., r_{|\mathcal{S}|}\right]$ is the vector whose generic element $r_{s}$ represents the bit rate assigned to source $s \in \mathcal{S}$;

- $\mathbf{p}=\left[p_{1}, p_{2}, . ., p_{j}, . ., p_{|\mathcal{N}|}\right]$ is the transmission power vector, where the generic element $p_{j}$ is the transmission power assigned to node $j \in \mathcal{N}$;

- $\mathbf{F}=\left[f_{i j}^{s}\right]$ is a binary matrix that represents the routing decisions, where the generic element $f_{i j}^{s}$ equals 1 iff link $(i, j)$ is part of the end-to-end path associated with source $s$;

- $\mathbf{P}^{e}=\left[P_{1}^{e}, P_{2}^{e}, . ., P_{j}^{e}, . ., P_{|\mathcal{N}|}^{e}\right]$ is a vector whose generic element $P_{j}^{e}$ represents the decoding error probability desired by node $j \in \mathcal{N}$;

$-d_{i j}()$ is the delay expression associated to link $(i, j)$, that models the specific physical and MAC layer, and their interaction with the routing and congestion control functions;

- $l_{i j}()$ is the capacity expression associated with link $(i, j)$, that depends on the physical layer characteristics;

$-B^{s}$ is the delay bound associated with source $s$;

$-\mathcal{U}_{s}$ and $\mathcal{V}_{j}$ are utility functions in the objective function, which model the desired optimality characteristics of the network, according to the application requirements.

The problem can be cast as follows:

\section{$\mathbf{P}^{O p t}$ : Cross-layer Resource Allocation}

$$
\begin{gathered}
\text { Given }: \quad P_{s}^{e}, d_{i j}(), l_{i j}(), B^{s} \\
\text { Find }: \quad \mathbf{r}, \mathbf{F}, \mathbf{p} \\
\text { Minimize }: \sum_{s \in \mathcal{S}} \mathcal{U}_{s}\left(r_{s}\right)+\sum_{j \in \mathcal{N}} \mathcal{V}_{j}\left(p_{j}\right) \\
\text { Subject to }: \\
\sum_{s \in \mathcal{S}} f_{i j}^{s} \cdot r_{s} \leq l_{i j}\left(\mathbf{P}^{e}, \mathbf{p}\right) ; \\
\sum_{(i, j) \in \mathcal{E}} f_{i j}^{s} \cdot d_{i j}\left(\mathbf{r}, l_{i j}\left(\mathbf{P}^{e}, \mathbf{p}\right)\right) \leq B_{s} ; \\
\mathbf{F} \in \mathcal{F}_{\text {feas }}(\mathbf{r}) ; \mathbf{r} \in \mathcal{R}_{\text {feas }} ; \mathbf{p} \in \mathcal{P}_{\text {feas }} .
\end{gathered}
$$

The above formulation jointly models problems at different layers in a crosslayer fashion. The optimization variables, whose values have to be jointly determined, are associated to different resources at different layers of the protocol stack. The transport problem consists of deciding the bit rate vector $\mathbf{r}$ to be assigned to the set of sources in the network. The routing problem consists of 
determining the routing matrix $\mathbf{F}$ according to which the sources route their data flows. The physical problem consists of selecting the optimal transmission power vector $\mathbf{p}$ that the set of sources should use. The above variables have to be jointly selected in order to maximize the objective function in (2). In particular, (2) maximizes the sum of the utilities of each source $s \in \mathcal{S}$ and of each node $j \in \mathcal{N}$, according to the utility functions $\mathcal{U}_{s}$ and $\mathcal{V}_{j}$, respectively. While the former increases with increasing bit rates granted to each source, the latter increases with decreasing power assigned to each node. Constraint (3) imposes that the resource utilized on each link be lower than the link capacity, which depends on the desired decoding error probability vector and on the used transmission powers. Constraint (4) forces the end-to-end delay of each source to be bounded by the maximum tolerated delay. The delay on each link can be expressed as a function of the assigned vector rate and link capacity. Constraints (5) impose limitations on the routing decisions, the available bit rates, and the selectable transmission powers, respectively, considering the MAC and physical constraints. Specifically, in the routing decision an end-to-end path is considered feasible if it is composed only of links connecting adjacent nodes. Moreover, concurrent transmissions are considered feasible if the generated interference is within certain bounds.

\section{Open Research Problems}

As explained in Sections 2 and 3, there exists some research on cross-layer interactions and design in developing new communication protocols. However, there is still much to be gained by rethinking the protocol functions of network layers in a unified way so as to provide a single communication module for efficient communication in WSNs. In other words, the cross-layer approach emerged recently, still necessitates a unified cross-layer communication protocol for efficient and reliable event communication that considers transport, routing, and medium access functionalities with physical layer (wireless channel) effects for WSNs.

There are several open research problems toward the development of systematic techniques for cross-layer design of wireless sensor network protocols:

Identify Adequate Utility Functions: A thorough study is needed to identify utility functions that: i) represent the desired global design objectives of sensor networks, such as minimal energy consumption and maximum network lifetime; ii) exhibit particular properties, e.g., convexity, that allow finding a unique global optimum with efficient methods and developing distributed implementations.

Improved Understanding of Energy Consumption: Existing studies on cross-layer optimization are mostly focused on jointly optimizing functionalities at different layers, usually with the overall objective of maximizing the network throughput. Conversely, in WSNs the ultimate objective is usually to minimize the energy consumption and/or to maximize the network lifetime. Hence, further study is needed to develop models and methodologies suitable to solve energyoriented problems. 
Accurate Delay Modeling: There is a need to develop sound models to include in the above framework an accurate description of the end-to-end delay as results from the interaction of the different layers. This is particularly important for the design of sensor network protocols for monitoring applications that require realtime delivery of event data, such as those encountered in wireless sensor and actor networks (WSAN) [2].

Connectivity with Realistic Physical Layer: Connectivity in wireless networks has been previously studied [16] [4, i.e., stochastic models have been developed to determine conditions under which a network is connected. These results, however, cannot be straightforwardly used, as they are based on the so-called unit disk graph communication model. However, recent experimental studies have demonstrated that the effects of the impairments of the wireless channel on higher-layer protocols are not negligible, as the availability of links further fluctuates because of channel fading phenomena that affect the wireless transmission medium. Furthermore, mobility of nodes is not considered. In fact, due to node mobility and node join and leave events, the network may be subject to frequent topological reconfigurations. Thus, links are continuously established and broken. For the above reasons, new analytical models are required to determine connectivity conditions that incorporate mobility and fading channels.

Cross-Layer Network Simulators: Current discrete-event network simulators such as OPNET [39], NS-2 [40, J-Sim [41], GloMoSim 42] may be unsuitable to implement a cross-layer solution, since their inner structure is based on a layered architecture, and each implemented functionality run by the simulator engine is tightly tied to this architecture. Hence, implementing a cross-layer solution in one of these simulators may turn into a non-trivial task. For this reason, there is a need to develop new software simulators that are based on a new developing paradigm so as to ease the development and test of cross-layer algorithmic and protocol solutions.

\section{Precautionary Guidelines in Cross-Layer Design}

In Section 4, we described several open research problems toward the development of systematic techniques for cross-layer design of wireless sensor network protocols. In this section, we describe possible risks raising when a cross-layer approach is followed, and propose precautionary guidelines and principles for cross-layer design beyond the open research issues presented in Section 4.

As stressed in Sections 2 and 3, the increased interactions and dependencies across layers turn into an interesting optimization opportunity that may be worth exploiting. Following this intuition, many cross-layer design papers that explore a much richer interaction between parameters across layers have been proposed in the recent past. While, however, as an immediate outcome most of these crosslayer suggestions may yield a performance improvement in terms of throughput or delay, this result is often obtained by decreasing the architecture modularity, and by loosing the logical separation between designers and developers. This 
abstraction decoupling is needed to allow the former to understand the overall system, and the latter to realize a more efficient production. For these reasons, when a cross-layer solution is proposed, the system performance gain needs to be weighed against the possible longer-term downfalls raised by a diminished degree of modularity.

In [19, the authors reexamine holistically the issue of cross-layer design and its architectural ramifications. They contend that a good architectural design leads to proliferation and longevity of a technology, and illustrate this with some historical examples, e.g., John von Neumann's architecture for computer systems, at the origin of the separation of software and hardware; the layered OSI architecture for networking, base of the current Internet architecture success; Shannon's architecture for communication systems, motivating the nonobvious separation of source and channel coding; last but not least, the plant controller feedback paradigm in control systems, providing universal principles common to human engineered systems as well as biological systems.

Although the concerns and cautionary advice expressed in [19] about crosslayer design are sound and well motivated, the layered-architecture, which turned to be a successful design choice for wired networks, may need to be carefully rethought for energy-constrained WSNs, where the concept itself of 'link' is labile, and many different effective transmission schemes and communication paradigms are conceivable.

This is also the conclusion drawn in [29, where the pros and cons of crosslayer design approach are evaluated. In [29, cross-layer design to improve reliability and optimize performance is advocated, although the design needs to be cautiously developed to provide long-term survivability of cross-layer architectures. In the following, we present some concerns and precautionary considerations, which need to be considered when a cross-layer design architecture is proposed, and suggest some possible research directions.

Modularity: In the classical layered design approach, a system architecture is broken down into modular components, and the interactions and dependencies between these components are systematically specified. This design philosophy allows to break complex problems into easier subproblems, which can then be solved in isolation, without considering all the details pertaining the overall system. This approach guarantees the inter-operability of subsystems in the overall system once each subsystem is tested and standardized, leading to quick proliferation of technology and mass production. Conversely, a cross-layer design approach may loose the decoupling between design and development process, which may impair both the design and the implementation development and slow the innovation down.

System Enhancement: Design improvements and innovations may become difficult in a cross-layer design, since it will be hard to assess how a new modification will interact with the already existing solutions. Furthermore, a cross-layer architecture would be hard to upkeep, and the maintaining costs would be high. In the worst cases, rather than modifying just one subsystem, the entire system 
may need to be replaced. For these reasons, we advocate keeping some degree of modularity in the design of cross-layer solutions. This could be achieved by relying on functional entities - as opposed to layers in the classical design philosophy - that implement particular functions. This would also have the positive consequence of limiting the duplication of functions that often characterizes a layered design. This functional redundancy is, in fact, one the cause for poor system performance.

Instability: In cross-layer design, the effect of any single design choice may affect the whole system, leading to various negative consequences such as instability. This is a non trivial problem to solve, since it is well known from control theory that stability is a paramount issue. Moreover, the fact that some interactions are not easily foreseen makes cross-layer design choices even trickier. Hence, great care should be paid to prevent design choices from negatively affecting the overall system performance. To this purpose, there is a need to integrate and further develop control theory techniques to study stability properties of system designed following a cross-layer approach. Dependency graphs, which may be used to capture the dependency relation between parameters, could be valuable means to prove stability, although hard to implement in some cases.

\section{Conclusions}

In this paper, we reviewed and classified literature on cross-layer protocols, improvements, and design methodologies for wireless sensor networks (WSNs). We overviewed the communication protocols devised for WSNs that focus on crosslayer design techniques. We classified these techniques based on the network layers they aim at replacing in the classical OSI network stack. Furthermore, we discussed systematic methodologies for the design of cross-layer solution for sensor networks as resource allocation problems in the framework of non-linear optimization. We outlined open research issues in the development of cross-layer methodologies for sensor networks and discussed possible research directions.

A cross-layer design methodology for energy-constrained wireless sensor networks is an appealing approach as long as cross-layer interactions are thoroughly studied and controlled. As pointed out in this paper, in fact, no cross-layer dependency should be left unintended, since this may lead to poor performance of the entire system.

\section{References}

1. I. F. Akyildiz, W. Su, Y. Sankarasubramaniam, Y., and E. Cayirci, "Wireless Sensor Networks: A Survey", Computer Networks (Elsevier) Journal, Vol. 38, No. 4, pp. 393-422, March 2002.

2. I. F. Akyildiz, and I. H. Kasimoglu, "Wireless Sensor and Actor Networks: Research Challenges," Ad Hoc Networks Journal (Elsevier), Vol. 2, No. 4, pp. 351-367, October 2004 .

3. I. F. Akyildiz, M. C. Vuran, and O. B. Akan, "Cross-Layer Module (XLM) for Wireless Sensor Networks," to appear in CISS '06, March 2006. 
4. Christian Bettstetter, "On the Minimum Node Degree and Connectivity of a Wireless Multihop Network," In Proc. ACM Intern. Symp. on Mobile Ad Hoc Networking and Computing (MobiHoc), Lausanne, Switzerland, pp. 80-91, June 9-11, 2002

5. Stephen Boyd, and Lieven Vandenberghe, "Convex Optimization," Cambridge University Press, March 2004.

6. J. H. Chang, and L. Tassiulas, "Energy conserving routing in wireless ad hoc networks," in Proc. IEEE INFOCOM 2000, March 2000, pp. 22-31.

7. L. Chen, S. H. Low, M. Chiang, and J. C. Doyle, "Optimal Cross-layer Congestion Control, Routing and Scheduling Design in Ad Hoc Wireless Networks, " submitted for publication.

8. M. Chiang, "Balancing transport and physical Layers in wireless multihop networks: jointly optimal congestion control and power control," IEEE JSAC, vol. 23, no. 1, pp. 104 116, Jan. 2005.

9. M. Chiang, "Geometric programming for communication systems", Short monograph in Foundations and Trends in Communications and Information Theory, vol. 2, no. 1-2, pp. 1-154, July 2005.

10. R. Cruz and A. Santhanam, "Optimal routing, link scheduling and power control in multi-hop wireless networks," in Proceedings of INFOCOM'03, San Francisco, USA, Mar. 2003, pp. 702-711.

11. S. Cui, R. Madan, A. Goldsmith, S. Lall, "Joint routing, MAC, and link layer optimization in sensor networks with energy constraints," in Proc. IEEE ICC '05, vol. 2, pp. 725 - 729, May 2005.

12. T. ElBatt and A. Ephremides, "Joint Scheduling and Power Control for Wireless Ad-hoc Networks," in Proceedings of INFOCOM'02, New York, June 2002.

13. Fang, Y., McDonald, B., "Dynamic codeword routing (DCR): a cross-layer approach for performance enhancement of general multi-hop wireless routing," 2004.

14. D. Ferrara, et. al., "MACRO: An Integrated MAC/Routing Protocol for Geographical Forwarding in Wireless Sensor Networks," in Proc. IEEE Infocom '05, vol. 3, pp. 1770 - 1781, March 2005.

15. D. Ganesan, et. al., "An empirical study of epidemic algorithms in large scale multihop wireless networks," Technical Report, Intel Research, 2002.

16. Gupta, P. and Kumar P. R., "Critical power for asymptotic connectivity in wireless networks," Stochastic Analysis, Control, Optimization and Applications: a volume in honor of W. H. Fleming, W. M. McEneaney, G. Yin and Q. Zhang, Birkhauser, Boston, 1998.

17. J. Haapola, Z. Shelby, C. Pomalaza-Raez, P. Mahonen, "Cross-layer energy analysis of multi-hop wireless sensor networks," in EWSN '05, pp. 33 - 44, 2005.

18. L. van Hoesel, T. Nieberg, J. Wu, and P. J. M. Havinga, "Prolonging the lifetime of wireless sensor networks by cross-layer interaction," IEEE Wireless Communications, vol. 11, no. 6, pp. 78 - 86, Dec. 2004.

19. V. Kawadia and P.R. Kumar, "A Cautionary Perspective On Cross-Layer Design," IEEE Wireless Communications Magazine, February 2005.

20. U. C. Kozat, I. Koutsopoulos, and L. Tassiulas, "A Framework for Cross-layer Design of Energy-efficient Communication with QoS Provisioning in Multi-hop Wireless Networks," in Proceedings of INFOCOM'04, Honk Kong, Mar. 2004.

21. S. H. Low, "A Duality Model of TCP and Queue Management Algorithms," IEEE/ACM Transactions on Networking, vol 11, no. 4, pp. 525-526, August 2003.

22. R. Madan, S. Cui; S. Lall, A. Goldsmith, "Cross-layer design for lifetime maximization in interference-limited wireless sensor networks," in Proc. IEEE INFOCOM '05, vol. 3, pp. 1964 - 1975, March 2005. 
23. T. Melodia, D. Pompili, and I. F. Akyildiz, "On the interdependence of distributed topology control and geographical routing in ad hoc and sensor networks," Journal of Selected Areas in Communications, vol. 23, no. 3, pp. 520-532, Mar. 2005.

24. Bozidar Radunovic, Jean-Yves Le Boudec, "Rate Performance Objectives of Multihop Wireless Networks," IEEE Transactions on Mobile Computing, vol. 03, no. 4, pp. 334-349, October 2004.

25. K. Seada, M. Zuniga, A. Helmy, B. Krishnamachari, "Energy-efficient forwarding strategies for geographic routing in lossy wireless sensor networks," in ACM Sensys '04, November 2004.

26. E. Shih, et. al., "Physical Layer Driven Protocol and Algorithm Design for EnergyEfficient Wireless Sensor Networks," in Proc. ACM MOBICOM '01, July 2001.

27. M. L. Sichitiu, "Cross-layer scheduling for power efficiency in wireless sensor networks," 2004.

28. P. Skraba, H. Aghajan, A. Bahai, "Cross-layer optimization for high density sensor networks: Distributed passive routing Decisions," in Proc. Ad-Hoc Now'04, Vancouver, July 2004.

29. S. Toumpis and A.J. Goldsmith, "Performance, Optimization, and Cross-Layer Design of Media Access Protocols for Wireless Ad Hoc Networks," in Proc. ICC, Seattle, Washington, USA, May 2003.

30. M. C. Vuran, O. B. Akan, and I. F. Akyildiz, "Spatio-Temporal Correlation: Theory and Applications for Wireless Sensor Networks," Computer Networks Journal (Elsevier), Vol. 45, No. 3, pp. 245-261, June 2004.

31. M. C. Vuran, V. B. Gungor, and O. B. Akan, "On the interdependency of congestion and contention in wireless sensor networks," in Proc. SENMETRICS '05, July 2005.

32. M. C. Vuran, and I. F. Akyildiz, "Spatial Correlation-based Collaborative Medium Access Control in Wireless Sensor Networks," to appear in IEEE/ACM Transactions on Networking, June 2006.

33. W. Yu and Jun Yuan, "Joint Source Coding, Routing, and Resource Allocation for Wireless Sensor Networks," in Proc. of IEEE International Conference on Communications (ICC), May 2005.

34. J. Yuan, Z. Li, W. Yu, B. Li, "A Cross-Layer optimization framework for multicast in multi-hop wireless networks wireless internet," in Proc. WICON '05, pp. 47 54, July 2005.

35. M. Zorzi, R. Rao, "Geographic random forwarding (GeRaF) for ad hoc and sensor networks: multihop performance," IEEE Trans. Mobile Computing, vol. 2, no. 4, pp. 337- 348, Oct.-Dec. 2003.

36. M. Zorzi, R. Rao, "Geographic random forwarding (GeRaF) for ad hoc and sensor networks: energy and latency performance," IEEE Trans. Mobile Computing, vol. 2, no. 4, pp. 349 365, Oct.-Dec. 2003.

37. M. Zorzi, "A new contention-based MAC protocol for geographic forwarding in ad hoc and sensor networks," in Proc. IEEE International Conference on Communications, vol. 6, pp. 3481 - 3485, June 2004.

38. M. Zuniga, B. Krishnamachari, "Analyzing the transitional region in low power wireless links," in Proc. IEEE SECON '04, pp. 517 - 526, Oct. 2004.

39. OPNET, http://www.opnet.com/

40. NS-2, http://www.isi.edu/nsnam/ns/

41. J-Sim, http://www.j-sim.org/

42. GloMoSim, http://pcl.cs.ucla.edu/projects/glomosim/ 